\title{
Rough Sets in Approximate Spatial Reasoning
}

\author{
Thomas Bittner and John G. Stell \\ 1 Centre de recherche en geomatique, Laval University, Quebec, Canada. \\ Thomas.Bittner@scg.ulaval.ca \\ 2 Department of Computer Science, Keele University, UK. john@ cs.keele.ac.uk
}

\begin{abstract}
In spatial reasoning the qualitative description of relations between spatial regions is of practical importance and has been widely studied. Examples of such relations are that two regions may meet only at their boundaries or that one region is a proper part of another. This paper shows how systems of relations between regions can be extended from precisely known regions to approximate ones. One way of approximating regions with respect to a partition of the plane is that provided by rough set theory for approximating subsets of a set. Relations between regions approximated in this way can be described by an extension of the RCC5 system of relations for precise regions. Two techniques for extending RC$\mathrm{C} 5$ are presented, and the equivalence between them is proved. A more elaborate approximation technique for regions (boundary sensitive approximation) takes account of some of the topological structure of regions. Using this technique, an extension to the RCC8 system of spatial relations is presented.
\end{abstract}

Keywords: qualitative spatial reasoning, approximate regions.

\section{Introduction}

Rough set theory [Paw91] provides a way of approximating subsets of a set when the set is equipped with a partition or equivalence relation. Given a set $X$ with a partition $\left\{a_{i} \mid i \in \mathcal{I}\right\}$, an arbitrary subset $b \subseteq X$ can be approximated by a function $\varphi_{b}: \mathcal{I} \rightarrow$ $\{\mathrm{fo}, \mathrm{po}, \mathrm{no}\}$. The value of $\varphi_{b}(i)$ is defined to be fo if $a_{i} \subseteq b$, it is no if $a_{i} \cap b=\varnothing$, and otherwise the value is po. The three values fo, po, and no stand respectively for 'full overlap', 'partial overlap' and 'no overlap'; they measure the extent to which $b$ overlaps the elements of the partition of $X$.

In spatial reasoning it is often necessary to approximate not subsets of an arbitrary set, but parts of a set with topological or geometric structure. For example the set $X$ above might be replaced by a regular closed subset of the plane, and we might want to approximate regular closed subsets of $X$. This approximation might be with respect to a partition of $X$ where the cells (elements of the partition) might overlap on their boundaries, but not their interiors. Because of the additional topological structure, it is possible to make a more detailed classification of overlaps between subsets and cells in the partition. An account of how this can be done was given in our earlier paper [BS98]. This is, however, only one of the directions in which the basic rough sets approach to approximation can be generalized to spatial approximation. 
Our concern in the present paper is relationships between spatial regions when these regions have been given approximate descriptions. The study of relationships between spatial regions is of practical importance in Geographic Information Systems (GIS), and has resulted in many papers [EF91,RCC92,SP92,Ben96]. Examples of relationships might be that two regions meet only at their boundaries or that one region is a proper part of another. While such relationships have been widely studied, the topic of relationships between approximate regions has received little attention.

The structure of the paper is as follows. In section 2 we set out the particular type of approximate regions we use in the main part of the paper. In section 3 we discuss one particular scheme of relationships between regions, known as the RCC5, and in section 4 we show how this can be generalized to approximate regions. In section 5 we briefly consider how our work can be extended to deal with more detailed boundary-sensitive approximations and the RCC8 system of relationships between regions. Finally in section 6 we present conclusions and suggest directions for further work.

\section{Approximating regions}

Spatial regions can be described by specifying how they relate to a frame of reference. In the case of two-dimensional regions, the frame of reference could be a partition of the plane into cells which may share boundaries but which do not overlap. A region can then be described by giving the relationship between the region and each cell.

\subsection{Boundary insensitive approximation}

Approximation functions Suppose a space $R$ of detailed or precise regions. By imposing a partition, $G$, on $R$ we can approximate elements of $R$ by elements of $\Omega_{3}^{G}$. That is, we approximate regions in $R$ by functions from $G$ to the set $\Omega_{3}=\{$ fo, po, no\}. The function which assigns to each region $r \in R$ its approximation will be denoted $\alpha_{3}: R \rightarrow \Omega_{3}^{G}$. The value of $\left(\alpha_{3} r\right) g$ is fo if $r$ covers all the of the cell $g$, it is po if $r$ covers some but not all of the interior of $g$, and it is no if there is no overlap between $r$ and $g$. We call the elements of $\Omega_{3}^{G}$ the boundary insensitive approximations of regions $r \in R$ with respect to the underlying regional partition $G$.

Each approximate region $X \in \Omega_{3}^{G}$ stands for a set of precise regions, i.e. all those precise regions having the approximation $X$. This set which will be denoted $\llbracket X \rrbracket$ provides a semantics for approximate regions: $\llbracket X \rrbracket=\left\{r \in R \mid \alpha_{3} r=X\right\}$.

Operations on approximation functions The domain of regions is equiped with a meet operation interpreted as the intersection of regions. In the domain of approximation functions the meet operation between regions is approximated by pairs of greatest minimal, $\wedge_{\min }$, and least maximal, $\wedge_{\max }$, meet operations on approximation mappings [BS98].

Consider the operations $\wedge_{\min }$ and $\wedge_{\max }$ on the set $\Omega_{3}=\{\mathrm{fo}, \mathrm{po}, \mathrm{no}\}$ that are defined as follows. 

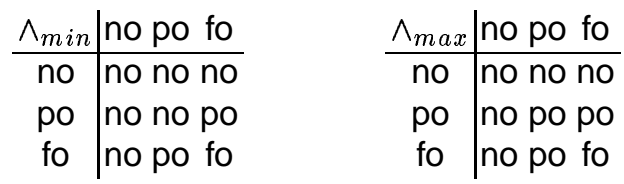

These operations extend to elements of $\Omega_{3}^{G}$ (i.e. the set of functions from $G$ to $\Omega_{3}$ ) by

$$
\left(X \wedge_{\min } Y\right) g=(X g) \wedge_{\min }(Y g)
$$

and similarly for $\wedge_{\max }$. This definition of the operations on $\Omega_{3}^{G}$ is equivalent to the construction for operations given by Bittner and Stell [BS98, page 108].

\subsection{Boundary sensitive approximation}

We can further refine the approximation of regions $R$ with respect to the partition $G$ by taking boundary segments shared by neighboring partition cells into account. That is, we approximate regions in $R$ by functions from $G \times G$ to the set $\Omega_{5}=$ $\{\mathrm{fo}, \mathrm{fbo}, \mathrm{pbo}, \mathrm{nbo}, \mathrm{no}\}$. The function which assigns to each region $r \in R$ its boundary sensitive approximation will be denoted $\alpha_{5}: R \rightarrow \Omega_{5}^{G \times G}$. The value of $\left(\alpha_{5} r\right)\left(g_{i}, g_{j}\right)$ is fo if $r$ covers all of the cell $g_{i}$, it is fbo if $r$ covers all of the boundary segment, $\left(g_{i}, g_{j}\right)$, shared by the cell $g_{i}$ and $g_{j}$ and some but not all of the interior of $g_{i}$, it is pbo if $r$ covers some but not all of the boundary segment $\left(g_{i}, g_{j}\right)$ and some but not all of the interior of $g_{i}$, it is nbo if $r$ does not intersect with boundary segment $\left(g_{i}, g_{j}\right)$ and some but not all of the interior of $g_{i}$, and it is no if there is no overlap between $r$ and $g_{i}$.

Let $b s=\left(g_{i} \cap g_{j}\right)$ be the boundary segment shared by the cell $g_{i}$ and $g_{j}$. We define boundary sensitive approximation in terms of pairs of approximation functions, $\alpha_{3}$, as follows [BS98]:

\begin{tabular}{l|ccc}
$\left(\alpha_{5} r\right)\left(g_{i}, g_{j}\right)=$ & $\left(\alpha_{3} r\right) b s=$ fo $\left(\alpha_{3} r\right) b s=$ po $\left(\alpha_{3} r\right) b s=$ no \\
\hline$\left(\alpha_{3} r\right) g_{i}=$ fo & fo & - & - \\
$\left(\alpha_{3} r\right) g_{i}=$ po & fbo & pbo & nbo \\
$\left(\alpha_{3} r\right) g_{i}=$ no & - & - & no
\end{tabular}

Each approximate region $X \in \Omega_{3}^{G \times G}$ stands for a set of precise regions, i.e. all those precise regions having the approximation $X: \llbracket X \rrbracket=\left\{r \in R \mid \alpha_{5} r=X\right\}$.

We define the operation $\wedge_{\max }$ on the set $\Omega_{5}=\{$ fo, fbo, pbo, nbo, no $\}$ as:

\begin{tabular}{|c||c|c|c|c|c|}
\hline$\wedge_{\max }$ & no & nbo & pbo & fbo & fo \\
\hline \hline no & no & no & no & no & no \\
\hline nbo & no & nbo & nbo & nbo & nbo \\
\hline pbo & no & nbo & pbo & pbo & pbo \\
\hline fbo & no & nbo & pbo & fbo & fbo \\
\hline fo & no & nbo & pbo & fbo & fo \\
\hline
\end{tabular}

This operation extends to elements of $\Omega_{5}^{G \times G}$ (i.e. the set of functions from $G \times G$ to $\left.\Omega_{5}\right)$ by $\left(X \wedge_{\max } Y\right)\left(g_{i}, g_{j}\right)=\left(X\left(g_{i}, g_{j}\right)\right) \wedge_{\max }\left(Y\left(g_{i}, g_{j}\right)\right)$. The definition of the operation $\wedge_{\min }$ is similar but silightly more complicated the details can be found in [BS98]. 


\section{RCC5 relations}

Qualitative spatial reasoning (QSR) is a well-established subfield of AI. It is concerned with the representation and processing of knowledge of space and activities which depend on space. However, the representations used for this are qualitative, rather than the quantitative ones of conventional coordinate geometry. One of the most widely studied formal approaches to QSR is the Region-Connection Calculus (RCC) [CBGG97]. This system provides an axiomatization of space in which regions themselves are primitives, rather than being constructed from more primitive sets of points. An important aspect of the body of work on RCC is the treatment of relations between regions. For example two regions could be overlapping, or perhaps only touch at their boundaries. There are two principal schemes of relations between RCC regions: five boundary insensitive relations known as RCC5, and eight boundary sensitive relations known as RCC8.

In this paper we propose a specific style of defining RCC relations. This style allows to define RCC relations exclusively based on constraints regarding the outcome of the meet operation between (one and two dimensional) regions. Furthermore this style of definitions allows us to obtain a partial ordering with minimal and maximal element on the relations defined. Both aspects are critical for the generalization of these relations to the approximation case.

Given two regions $x$ and $y$ the RCC5 relation between them can be determined by considering the triple of boolean values:

$$
(x \wedge y \neq \perp, x \wedge y=x, x \wedge y=y) .
$$

The correspondence between such triples and the RCC5 classification is given in the following table. Possible geometric interpretations are given in figure 1.

\begin{tabular}{|c|c|c|c|}
\hline$x \wedge y \neq \perp$ & $x \wedge y=x$ & $x \wedge y=y$ & RCC5 \\
\hline $\mathrm{F}$ & $\mathrm{F}$ & $F$ & \begin{tabular}{|l|} 
DR \\
\end{tabular} \\
\hline $\mathrm{T}$ & $\mathrm{F}$ & $\mathrm{F}$ & $\mathrm{PO}$ \\
\hline $\mathrm{T}$ & $\mathrm{T}$ & $\mathrm{F}$ & PP \\
\hline $\mathrm{T}$ & $\mathrm{F}$ & $\mathrm{T}$ & PPi \\
\hline $\mathrm{T}$ & T & T & $E Q$ \\
\hline
\end{tabular}

The set of triples is partially ordered by setting $\left(a_{1}, a_{2}, a_{3}\right) \leq\left(b_{1}, b_{2}, b_{3}\right)$ iff $a_{i} \leq b_{i}$ for $i=1,2,3$, where the Boolean values are ordered by $\mathrm{F}<\mathrm{T}$. This is the same ordering induced by the RCC5 conceptual graph [GC94]. But note that the conceptual graph has $P O$ and $E Q$ as neighbours which is not the case in the Hasse diagram for the partially ordered set (Right diagram in figure 1). We refer to this as the RCC5 lattice to distinguish it from the conceptual neighborhood graph.

\section{Semantic and Syntactic Generalizations of RCC5}

The original formulation of RCC dealt with ideal regions which did not suffer from imperfections such as vagueness, indeterminacy or limited resolution. However, these are factors which affect spatial data in practical examples, and which are significant in 


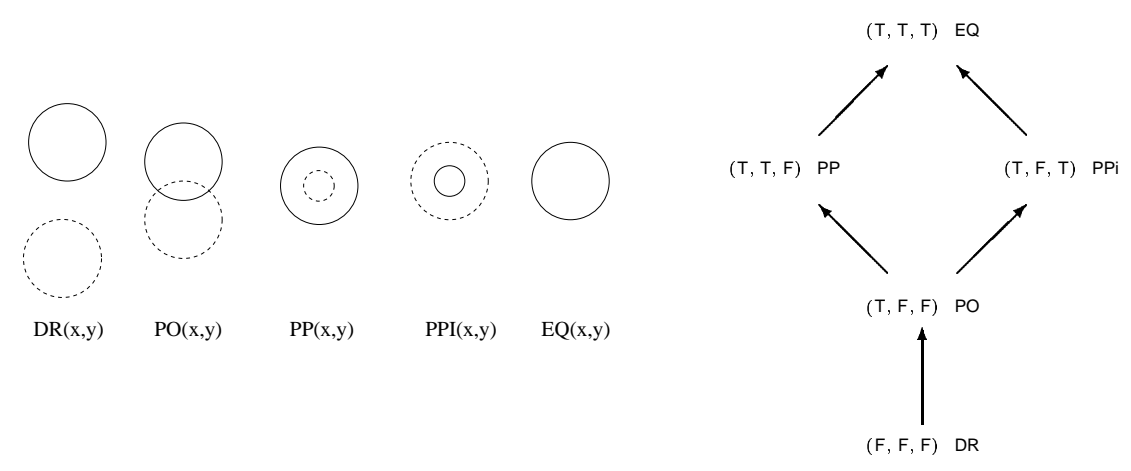

Fig. 1. RCC5 relations and RCC5 lattice

applications such as geographic information systems (GIS)[BF95]. The issue of vagueness and indeterminacy has been tackled in the work of [CG96]. The topic of the present paper is not vagueness or indeterminacy in the widest sense, but rather the special case where spatial data is approximated by being given a limited resolution description.

\subsection{Syntactic and semantic generalizations}

There are two approaches we can take to generalizing the RCC5 classification from precise regions to approximate ones. These two may be called the semantic and the syntactic. The syntactic has many variants.

Semantic We can define the RCC5 relationship between approximate regions $X$ and $Y$ to be the set of relationships which occur between any pair of precise regions representing $X$ and $Y$. That is, we can define

$$
\operatorname{SE\mathcal {N}}(X, Y)=\{R C C 5(x, y) \mid x \in \llbracket X \rrbracket \text { and } y \in \llbracket Y \rrbracket\} .
$$

Syntactic We can take a formal definition of RCC5 in the precise case which uses operations on $R$ and generalize this to work with approximate regions by replacing the operations on $R$ by analogous ones for $\Omega^{G 1}$.

\subsection{Syntactic generalization}

The above formulation of the RCC5 relations can be extended to approximate regions. One way to do this is to replace the operation $\wedge$ with an appropriate operation for

\footnotetext{
${ }^{1}$ This technique has many variants since there are many different ways in which the RCC5 can be formally defined in the precise case, and some of these can be generalized in different ways to the approximate case. The fact that several different generalizations can arise from the same formula is because some of the operations in $R$ (such as $\wedge$ and $\vee$ ) have themselves more than one generalization to operations on $\Omega^{G}$.
} 
approximate regions. If $X$ and $Y$ are approximate regions (i.e. functions from $G$ to $\Omega_{3}$ ) we can consider the two triples of Boolean values:

$$
\begin{aligned}
& \left(X \wedge_{\text {min }} Y \neq \perp, X \wedge_{\text {min }} Y=X, X \wedge_{\text {min }} Y=Y\right), \\
& \left(X \wedge_{\text {max }} Y \neq \perp, X \wedge_{\text {max }} Y=X, X \wedge_{\text {max }} Y=Y\right) .
\end{aligned}
$$

In the context of approximate regions, the bottom element, $\perp$, is the function from $G$ to $\Omega_{3}$ which takes the value no for every element of $G$. Each of the above triples provides an RCC5 relation, so the relation between $X$ and $Y$ can be measured by a pair of RCC5 relations. These relations will be denoted by $R_{\min }(X, Y)$ and $R_{\max }(X, Y)$.

Theorem 1 The pairs $\left(R_{\min }(X, Y), R_{\max }(X, Y)\right)$ which can occur are all pairs $(a, b)$ where $a \leq b$ with the exception of (PP, EQ) and (PPi, EQ).

Proof First we show that $R_{\min }(X, Y) \leq R_{\max }(X, Y)$. Suppose that $R_{\min }(X, Y)=$ $\left(a_{1}, a_{2}, a_{3}\right)$ and that $R_{\max }(X, Y)=\left(b_{1}, b_{2}, b_{3}\right)$. We have to show that $a_{i} \leq b_{i}$ for $i=1,2,3$. Taking the first component, if $X \wedge_{\min } Y \neq \perp$ then for each $g$ such that $X g \wedge_{\min } Y g \neq$ no, we also have, by examining the tables for $\wedge_{\min }$ and $\wedge_{\max }$, that $X g \wedge_{\max } Y g \neq$ no. Hence $X \wedge_{\max } Y \neq \perp$. Taking the second component, if $X \wedge_{\min } Y=X$ then $X \wedge_{\max } Y=X$ because from $X g \wedge_{\text {min }} Y g=X g$ it follows that $X g \wedge_{\max } Y g=X g$. This can be seen from the tables for $\wedge_{\min }$ and $\wedge_{\max }$ by considering each of the three possible values for $X g$. The case of the third component follows from the second since $\wedge_{\min }$ and $\wedge_{\max }$ are commutative.

Finally we have to show that the pairs (PP, EQ) and (PPi, EQ) cannot occur. If $R_{\max }(X, Y)=\mathrm{EQ}$, then $X=Y$ so $X \wedge_{\min } Y=X$ must take the same value as $X \wedge_{\min } Y=Y$. Thus the only triples which are possible for $R_{\min }(X, Y)$ are those where the second and third components are equal. This rules out the possibility that $R_{\min }(X, Y)$ is $\mathrm{PP}$ or PPi.

\subsection{Correspondence of semantic and syntactic generalization}

Let the syntactic generalization of RCC5 defined by

$$
\operatorname{sy\mathcal {N}}(X, Y)=\left(R_{\min }(X, Y), R_{\max }(X, Y)\right),
$$

where $R_{\min }$ and $R_{\max }$ are as defined above.

Theorem 2 For any approximate regions $X$ and $Y$, the two ways of measuring the relationship of $X$ to $Y$ are equivalent in the sense that

$$
\operatorname{SE\mathcal {N}}(X, Y)=\left\{\rho \in R C C 5 \mid R_{\min }(X, Y) \leq \rho \leq R_{\text {max }}(X, Y)\right\},
$$

where $R C C 5$ is the set $\{\mathrm{EQ}, \mathrm{PP}, \mathrm{PPi}, \mathrm{PO}, \mathrm{DR}\}$, and $\leq$ is the ordering in the $R C C 5$ lattice.

The proof of this theorem depends on assumptions about the set of precise regions $R$. We assume that $R$ is a model of the RCC axioms so that we are approximating continuous space, and not approximating a space of already approximated regions. 
Proof There are three things to demonstrate. Firstly that for all $x \in \llbracket X \rrbracket$, and $y \in \llbracket Y \rrbracket$, that $R_{\min }(X, Y) \leq R C C 5(x, y)$. Secondly, for all $x$ and $y$ as before, that $R C C 5(x, y) \leq R_{\max }(X, Y)$, and thirdly that if $\rho$ is any RCC5 relation such that $R_{\text {min }}(X, Y) \leq \rho \leq R_{\max }(X, Y)$ then there exist particular $x$ and $y$ which stand in the relation $\rho$ to each other. To prove the first of these it is necessary to consider each of the three components $X \wedge_{\text {min }} Y \neq \perp, X \wedge_{\text {min }} Y=X$ and $X \wedge_{\text {min }} Y=Y$ in turn. If $X \wedge_{\text {min }} Y \neq \perp$ is true, we have to show for all $x$ and $y$ that $x \wedge y \neq \perp$ is also true. From $X \wedge_{\min } Y \neq \perp$ it follows that there is at least one cell $g$ where one of $X$ and $Y$ fully overlaps $g$, and the other at least partially overlaps $g$. Hence there are interpretations of $X$ and $Y$ having non-empty intersection. If $X \wedge_{\min } Y=X$ is true then for all cells $g$ we have $X g=$ no or $Y g=$ fo. In each case every interpretation must satisfy $x \wedge y=x$. Note that this depends on the fact that the combination $X g=\mathrm{po}=Y g$ cannot occur. The case of the final component $X \wedge_{\min } Y=Y$ is similar. Thus we have demonstrated for all $x \in \llbracket X \rrbracket$ and $y \in \llbracket Y \rrbracket$ that $R_{\min }(X, Y) \leq R C C 5(x, y)$. The task of showing that $R C C 5(x, y) \leq R_{\max }(X, Y)$ is accomplished by a similar analysis. Finally, we have to show that for each RCC5 relation, $\rho$, where $R_{\min }(X, Y) \leq \rho \leq R_{\max }(X, Y)$, there are $x \in \llbracket X \rrbracket$ and $y \in \llbracket Y \rrbracket$ such that the relation of $x$ to $y$ is $\rho$. This is done by considering the various possibilities for $R_{\min }(X, Y)$ and $R_{\max }(X, Y)$. We will only consider one of the cases here, but the others are similar. If $R_{\min }(X, Y)=\mathrm{PO}$ and $R_{\text {max }}(X, Y)=\mathrm{EQ}$, then for each cell $g$, the values of $X g$ and $Y g$ are equal and there must be some cells where this value is po and some cells where the value is fo. Precise regions $x \in \llbracket X \rrbracket$ and $y \in \llbracket Y \rrbracket$ can be constructed by selecting sub-regions of each cell $g$ say $x_{g}$ and $y_{g}$, and defining $x$ and $y$ to be the unions of these sets of sub-regions. In this particular case, there is sufficient freedom with those cells where $X g=Y g=$ po to be able to select $x_{g}$ and $y_{g}$ so that the relation of $x$ to $y$ can be any $\rho$ where $\mathrm{PO} \leq \rho \leq \mathrm{EQ}$.

\section{Generalizing $\mathrm{RCC8}$ relations}

\subsection{RCC8 relations}

RCC8 relations take the topological distinction betwen interior and boundary into account. In order to describe RCC8 relations we define the relationship between $x$ and $y$ by using a triple, but where the three entries may take one of three truth values rather than the two Boolean ones. The scheme has the form

$$
(x \wedge y \not \approx \perp, x \wedge y \approx x, x \wedge y \approx y)
$$

where

$$
x \wedge y \not \perp=\left\{\begin{array}{c}
\mathrm{T} \text { if the interiors of } x \text { and } y \text { overlap, } \\
\text { i.e., } x \wedge y \neq \perp \\
\mathrm{M} \text { if only the boundaries } x \text { and } y \text { overlap } \\
\quad \text { i.e., } x \wedge y=\perp \text { and } \delta x \wedge \delta y \neq \perp \\
\mathrm{F} \text { if there is no overlap between } x \text { and } y \\
\text { i.e., } x \wedge y=\perp \text { and } \delta x \wedge \delta y=\perp
\end{array}\right.
$$


and where

$x \wedge y \approx x=\left\{\begin{array}{c}\mathrm{\top} \text { if } x \text { is contained in } y \text { and the boundaries are either disjoint or identical } \\ i . e ., x \wedge y=x \text { and }(\delta(x) \wedge \delta(y)=\perp \text { or } \delta(x) \wedge \delta(y)=\delta(x)) \\ \mathrm{M} \text { if } x \text { is contained in } y \text { and the boundaries are not disjoint and not identical, } \\ \text { i.e., } x \wedge y=x \text { and } \delta x \wedge \delta y \neq \perp \text { and } \delta(x) \wedge \delta(y) \neq \delta(x) \\ \mathrm{F} \text { if } x \text { is not contained within } y, \text { i.e., } x \wedge y \neq x\end{array}\right.$ and similarly for $x \wedge y \approx y$.

The correspondence between such triples and the RCC 8 classification is given in the following table. Possible geometric interpretations can be found in figure 2 .

\begin{tabular}{|c|c|c|c|}
\hline$x \wedge y \not \approx$ & $\perp \mid x \wedge y \approx x$ & $x \wedge y \approx y$ & \begin{tabular}{|l|} 
RCC 8 \\
\end{tabular} \\
\hline $\mathrm{F}$ & \begin{tabular}{l|l}
$F$ \\
\end{tabular} & $\mathrm{~F}$ & DC \\
\hline M & $\mathrm{F}$ & $\mathrm{F}$ & EC \\
\hline $\mathrm{T}$ & $\mathrm{F}$ & $\mathrm{F}$ & PO \\
\hline $\mathrm{T}$ & M & $\mathrm{F}$ & TPP \\
\hline $\mathrm{T}$ & $\mathrm{T}$ & $\mathrm{F}$ & NTPP \\
\hline $\mathrm{T}$ & $\mathrm{F}$ & M & TPPi \\
\hline $\mathrm{T}$ & $F$ & T & NTPPi \\
\hline $\mathrm{T}$ & $\mathrm{T}$ & $\mathrm{T}$ & EQ \\
\hline
\end{tabular}

The RCC5 relation DR refines to DC and EC and the RCC5 relation $\mathrm{PP}(i)$ refines to $\operatorname{TPP}(i)$ and $\operatorname{NTPP}(i)$. We define $\mathrm{F}<\mathrm{M}<\mathrm{T}$ and call the corresponding Hasse diagram RCC8 lattice (figure 2 to distinguish it from the conceptual neighborhood graph.

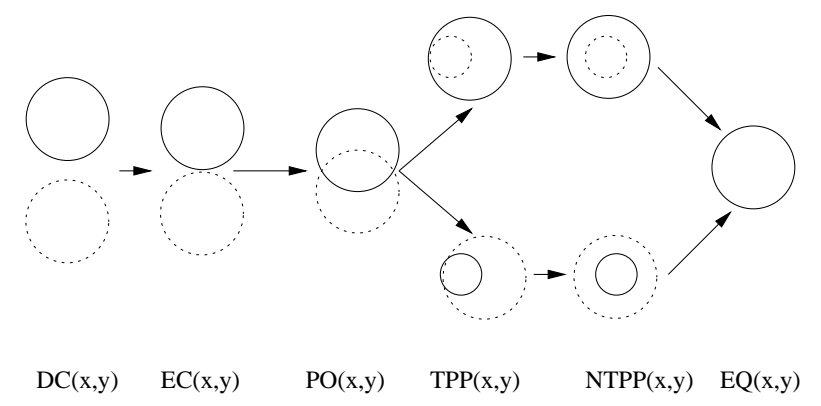

Fig. 2. RCC8 lattice

\subsection{Syntactic generalization of RCC8}

Let $X$ and $Y$ be boundary sensitive approximations of regions $x$ and $y$. The generalized scheme has the form

$$
\begin{aligned}
& \left(\left(X \wedge_{\min } Y \not \Perp \perp, X \wedge_{\min } Y \approx X, X \wedge_{\min } Y \approx Y\right),\right. \\
& \left.\left(X \wedge_{\max } Y \not \approx \perp, X \wedge_{\max } Y \approx X, X \wedge_{\max } Y \approx Y\right)\right)
\end{aligned}
$$


where

$$
X \wedge_{\text {min }} Y \not \perp=\left\{\begin{array}{l}
\mathrm{T} X \wedge_{\text {min }} Y \neq \perp \\
\mathrm{M} X \wedge_{\text {min }} Y=\perp \text { and } \delta X \wedge_{\text {min }} \delta Y \neq \perp \\
\mathrm{F} X \wedge_{\text {min }} Y=\perp \text { and } \delta X \wedge_{\text {min }} \delta Y=\perp
\end{array}\right.
$$

and where

$X \wedge_{\text {min }} Y \approx X=\left\{\begin{array}{l}\mathrm{T} X \wedge_{\text {min }} Y=X \text { and }\left(\delta X \wedge_{\text {min }} \delta Y=\perp \text { or } X \wedge_{\text {min }} Y=Y\right) \\ \mathrm{M} X \wedge_{\text {min }} Y=X \text { and } \delta X \wedge_{\text {min }} \delta Y \neq \perp \text { and } X \wedge_{\text {min }} Y \neq Y \\ \mathrm{~F} X \wedge_{\text {min }} Y \neq X\end{array}\right.$

and similarly for $X \wedge_{\min } Y \approx Y, X \wedge_{\max } Y \not \downarrow \perp, X \wedge_{\max } Y \approx X$, and $X \wedge_{\max } Y \approx Y$. In this context the bottom element, $\perp$, is the function from $G \times G$ to $\Omega_{5}$ which takes the value no for every element of $G \times G$. The formula $\delta X \wedge_{\min } \delta Y \neq \perp$ is true if we can derive from boundary sensitive approximations $X$ and $Y$ that for all $x \in \llbracket X \rrbracket$ and $y \in$ $\llbracket Y \rrbracket$ the least relation that can hold between $x$ and $y$ involves boundary intersection ${ }^{2}$. Correspondingly, $\delta X \wedge_{\max } \delta Y \neq \perp$ is true if the largest relation that can hold between $x \in \llbracket X \rrbracket$ and $y \in \llbracket Y \rrbracket$ involves boundary intersection.

Each of the above triples defines a RCC 8 relation, so the relation between $X$ and $Y$ can be measured by a pair of RCC8 relations. These relations will be denoted by $R_{\text {min }}^{8}(X, Y)$ and $R_{\text {max }}^{8}(X, Y)$. [BS00] show the correspondence between this syntactic generalization and the semantin generalization corresponding to the RCC5 case.

\section{Conclusions}

In this paper we discussed approximations of spatial regions with respect to an underlying regional partitions. We used approximations based on approximation functions and discussed the close relationship to Rough sets. We defined pairs of greatest minimal and least maximal meet operations on approximation functions that constrain the possible outcome of the meet operation between the approximated regions themselves. The meet operations on approximation mappings provide the basis for approximate qualitative spatial reasoning that was proposed in this paper.

Approximate qualitative spatial reasoning is based on:

1. Jointly exhaustive and pair-wise disjoint sets of qualitative relations between exact regions, which are defined in terms of the meet operation of the underlying Boolean algebra structure of the domain of regions. As a set these relations must form a lattice with bottom and top element.

2. Approximations of regions with respect to a regional partition of the underlying space. Semantically, an approximation corresponds to the set of regions it approximates.

3. Pairs of meet operations on those approximations, which approximate the meet operation on exact regions.

\footnotetext{
${ }^{2}$ For details see [BS00].
} 
Based on those 'ingredients' syntactic and semantic generalizations of jointly exhaustive and pair-wise disjoint relations between exact regions were defined. Generalized relations hold between approximations of regions rather than between (exact) regions themselves. Syntactic generalization is based on replacing the meet operation defining relations between exact regions by its minimal and maximal counterparts on approximations. Semantically, syntactic generalizations yield upper and lower bounds (within the underlying lattice structure) on relations that can hold between the corresponding approximated exact regions.

There is considerable scope for further work building on the results in this paper. We have assumed so far that the regions being approximated are precisely known regions in a continuous space. However, there are practical examples where approximate regions are themselves approximated. This can occur when spatial data is required at several levels of detail, and the less detailed representations are approximations of the more detailed ones. Thus one direction for future investigation is to extend the techniques in this paper to the case where the regions being approximated are discrete, rather than continuous. This could make use of the algebraic approach to qualitative discrete space presented in [?]. Subject of ongoing research is to apply techniques presented in this paper to the temporal domain [Bit00].

\section{References}

[Ben96] B. Bennett. An application of qualitative spatial reasoning to gis. In 1st. International Conference on GeoComputation, Leeds, 1996.

[BF95] Peter Burrough and Andrew U. Frank, editors. Geographic Objects with Indeterminate Boundaries. GISDATA Series II. Taylor and Francis, London, 1995.

[Bit00] T. Bittner. Approximate temporal reasoning. In Workshop proceedings of the Seventeenth National Conference on Artificial Intelligence, AAAI 2000, 2000.

[BS98] T. Bittner and J. G. Stell. A boundary-sensitive approach to qualitative location. Annals of Mathematics and Artificial Intelligence, 24:93-114, 1998.

[BS00] T. Bittner and J. Stell. Approximate qualitative spatial reasoning. Technical report, Department of Computing and Information Science, Queen's University, 2000.

[CBGG97] A.G. Cohn, B. Bennett, J. Goodday, and N. Gotts. Qualitative spatial representation and reasoning with the region connection calculus. geoinformatica, 1(3):1-44, 1997.

[CG96] A.G. Cohn and N.M. Gotts. The 'egg-yolk' representation of regions with indeterminate boundaries. In P. Burrough and A.U. Frank, editors, Geographic Objects with Indeterminate Boundaries, GISDATA Series II. Taylor and Francis, London, 1996.

[EF91] Max J. Egenhofer and Robert D. Franzosa. Point-set topological spatial relations. International Journal of Geographical Information Systems, 5(2):161-174, 1991.

[GC94] J.M. Goodday and A.G. Cohn. Conceptual neighborhoods in temporal and spatial reasoning. In ECAI-94 Spatial and Temporal Reasoning Workshop, 1994.

[Paw91] Zdzis aw Pawlak. Rough sets : theoretical aspects of reasoning about data. Theory and decision library. Series D, System theory, knowledge engineering, and problem solving ; v. 9. Kluwer Academic Publishers, Dordrecht ; Boston, 1991.

[RCC92] D. A. Randell, Z. Cui, and A. G. Cohn. A spatial logic based on regions and connection. In 3rd Int. Conference on Knowledge Representation and Reasoning. Boston, 1992.

[SP92] T.R. Smith and K. K. Park. Algebraic approach to spatial reasoning. Int. J. Geographical Information Systems, 6(3):177 - 192, 1992. 